\title{
Primeros registros de presencia de Nothofagus nitida (Nothofagaceae) en Argentina y estimación de su área de distribución potencial
}

\author{
First presence records of Nothofagus nitida (Nothofagaceae) \\ in Argentina and estimation of its potential distribution area
}

\author{
Paula Mathiasen a, Félix Vidoz ${ }^{\text {b }}$, Sebastián Valle c, Valeria S Ojeda a , María Cristina Acosta ${ }^{\text {d, }}$ \\ Silvina Ippi e , Gerardo A Cerón e, Andrea C Premoli a* \\ *Autor de correspondencia: ${ }^{a}$ Universidad Nacional del Comahue, INIBIOMA-CONICET, Quintral 1250, 8400 Bariloche, \\ Río Negro, Argentina, tel.: +54 2944428505 Int. 110, andrea.premoli@gmail.com

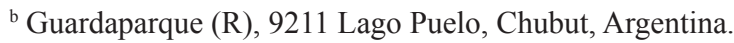 \\ ${ }^{\mathrm{c}}$ Cuerpo de Guardaparques, Parque Nacional Los Alerces, Administración de Parques Nacionales, \\ 9201 Villa Futalaufquen, Chubut, Argentina. \\ d Universidad Nacional de Córdoba, Instituto Multidisciplinario de Biología Vegetal (IMBIV)-CONICET, Córdoba, Argentina.

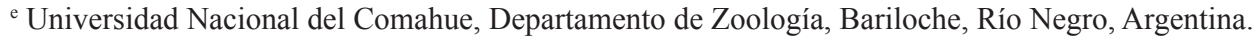

\begin{abstract}
SUMMARY
Nothofagus nitida is an evergreen tree species considered endemic to the humid forests of Southern Chile. In this study, we inform the finding of $N$. nitida individuals on the eastern slopes of the Andes in Argentina, expanding its potential geographic distribution range, restricted until now to humid areas west of the Andean range, in Chile. New localities correspond to the south outlet of Menéndez Lake on riverbanks of Río Alerce and Río del Paso Viejo, in Los Alerces National Park, Chubut Province, Argentina. Samples of fresh leaves were collected from five individuals that were considered as putative $N$. nitida, based on leaf and bark morphology. We performed automated sequencing of the ITS nuclear DNA region, which was previously used to identify different species of subgenus Nothofagus. Four of the obtained DNA sequences corresponded to $N$. nitida. In addition, we carried out a species distribution model that showed other potential areas of occurrence in Argentina. This finding merits the exploring efforts in other localities at the East of the Andean Range, using as guidance the potential distribution maps in order to determine its current distribution and genetic markers to confirm its presence.
\end{abstract}

Key words: Argentina, Chile, coihue de Chiloé, species potential distribution models, Nothofagus nitida, ITS DNA sequences.

\section{RESUMEN}

Nothofagus nitida es una especie arbórea siempreverde considerada endémica de los bosques húmedos del sur de Chile. En este estudio, se informa el hallazgo de individuos de $N$. nitida al este de Los Andes en Argentina, ampliándose su área de distribución geográfica potencial, hasta ahora documentada solo para zonas húmedas al oeste de la Cordillera de Los Andes, en Chile. Las nuevas localidades corresponden al Brazo Sur del Lago Menéndez a orillas de los ríos Alerce y del Paso Viejo, en el Parque Nacional Los Alerces, Provincia de Chubut, Argentina. Se tomaron muestras de follaje fresco de cinco individuos que fueron considerados $N$. nitida putativos a base de la morfología foliar y corteza. Se extrajo ADN, se amplificó mediante PCR y se secuenció de manera automática la región ITS del ADN nuclear que permite identificar las distintas especies del subgénero Nothofagus. Las secuencias de ADN obtenidas de cuatro individuos correspondieron de manera inequívoca a $N$. nitida. Por otro lado, se realizó un modelado de la distribución de la especie que mostró otras áreas potenciales de ocurrencia en Argentina. Este hallazgo amerita la realización de esfuerzos para su búsqueda en otras localidades al este de Los Andes, utilizando como herramienta los mapas de distribución potencial a fin de determinar su distribución y utilizar marcadores genéticos para confirmar su presencia.

Palabras clave: Argentina, Chile, coihue de Chiloé, modelos de distribución potencial de especies, Nothofagus nitida, secuencias ADN ITS

\section{INTRODUCCIÓN}

El subgénero Nothofagus consiste de cinco especies leñosas que solo habitan los Bosques Templados Austra- les de Argentina y Chile (Hill y Read 1991, Veblen et al. 1996). Dos de estas especies son deciduas o de hoja caduca y son conocidas comúnmente como ñire ( $N$. antarctica (G. Forst.) Oerst.), y lenga (N. pumilio (Poepp. et Endl.) 
Krasser); mientras que las otras tres son siempreverdes: coihue ( $N$. dombeyi (Mirb.) Oerst.), coihue de Magallanes o guindo ( $N$. betuloides (Mirb.) Oerst.), y coihue de Chiloé ( $N$. nitida (Phil.) Krasser). Excepto N. nitida todas las especies poseen relativamente amplios rangos latitudinales de distribución. Nothofagus nitida crece principalmente en condiciones de clima oceánico templado húmedo, ocupando los cinturones de alta precipitación en bosques de baja a mediana altitud (entre los 500 y 800 m s.n.m.). De las tres especies siempreverdes, $N$. nitida es la que presenta el rango de distribución más restringido principalmente en la Cordillera de la Costa y el archipiélago de Chiloé y en la Cordillera de Los Andes, desde los 39 $45^{\prime}$ a los $48^{\circ} 30^{\prime} \mathrm{S}$ (Veblen et al. 1996, Rodríguez y Quezada 2003, Donoso et al. 2004, Donoso y Escobar 2006). Hasta la fecha N. nitida ha sido documentada solo en Chile entre las provincias de Valdivia (entre los lagos Ranco y Puyehue) y Última Esperanza, en la Cordillera de Los Andes, y en la Cordillera de la Costa desde el sur del río Valdivia hasta el sector costero continental (Península de Taitao). En la Depresión Central se encuentra en las áreas de ñadis al norte del seno de Reloncaví, y en las áreas planas húmedas de Chiloé (Donoso et al. 2004), siempre al oeste de la Cordillera de Los Andes.

Nothofagus nitida es un árbol de gran tamaño que puede alcanzar hasta $35 \mathrm{~m}$ de altura y $2 \mathrm{~m}$ de diámetro (Rodríguez y Quezada 2003). Se distingue de las otras especies de Nothofagus siempreverdes en cuanto a su morfología: su corteza es gris oscura y más lisa que $N$. dombeyi y $N$. betuloides; sus hojas son coriáceas y de mayor tamaño, más oscuras, más brillantes y tienen forma trapezoidal-romboidal (Premoli 1996b). Sin embargo, $N$. dombeyi presenta una amplia variabilidad fenotípica foliar a lo largo de su rango de distribución geográfica, desde hojas casi redondeadas (similares a $N$. betuloides) hasta hojas trapezoidales-romboidales (tipo punta de flecha) similares a N. nitida (Premoli 1996b), lo que en varias ocasiones hace difícil la identificación de las especies basándose solo en la morfología foliar. Las flores masculinas se disponen de a tres sobre un pedúnculo común y las flores femeninas pueden ser tres a cinco por cúpula, a diferencia de $N$. dombeyi y $N$. betuloides que solo poseen tres ubicadas en una cúpula de cuatro valvas. Los miembros del subgénero Nothofagus, incluida $N$. nítida, son monoicos y comparten la dispersión del polen anemófila y una dispersión limitada de las semillas que ocurre en la cercanía del árbol semillero (Veblen et al. 1996). El fruto consiste en una cúpula que contiene tres nueces de 1-2 $\mathrm{mm}$ de largo, la central bialada y las laterales trialadas, bastante pequeñas de color café intenso (Rodríguez y Quezada 2003, Donoso y Escobar 2006). Debido a su distribución relativamente restringida, $N$. nitida posee una escasa variación intraespecífica, principalmente por las condiciones climáticas en que se encuentra (Piper et al. 2007).

La morfología foliar ha sido utilizada extensamente en Nothofagus spp. a fin de describir las especies actuales y extintas y para investigar las relaciones evolutivas entre los distintos taxones. Las tres especies siempreverdes del género Nothofagus han sido consideradas cercanamente emparentadas (Premoli 1996a, Acosta y Premoli 2010). El hecho de que compartan el mismo nombre común "coihue o coigüe", refleja su elevada similitud morfológica, lo que dificulta poder discernir entre ellas cuando ocurren en simpatría en bosques mixtos (Donoso et al. 2004). Por este motivo, es necesario recurrir a otro tipo de información para poder identificar certeramente a las especies. Tal es el caso de los marcadores moleculares los cuales se basan en el estudio de las características genéticas de los individuos, que son conservadas dentro de una misma especie. Se han realizado numerosos estudios con marcadores moleculares para resolver las relaciones evolutivas entre las especies del género Nothofagus (por ejemplo, Manos 1997). En particular, un estudio filogenético realizado con las especies pertenecientes a este subgénero utilizando marcadores moleculares del ADN nuclear (espaciador intergénico transcripto, ITS) permitió elucidar que $N$. pumilio divergió primeramente del resto, siendo $N$. antarctica la especie hermana del clado monofilético que contiene a las especies siempreverdes ( $N$. dombeyi, $N$. nitida y $N$. betuloides), dentro del cual $N$. nitida habría divergido más tempranamente (Premoli 1996a, Acosta y Premoli 2010). Estas relaciones filogenéticas fueron determinadas mediante secuencias de $\mathrm{ADN}$ de la región nuclear ITS que se puede considerar un carácter diagnóstico ya que permite identificar a las distintas especies del subgénero (Acosta y Premoli 2010). Estudios genético-poblacionales de las tres especies siempreverdes de Nothofagus, mostraron una asociación positiva entre el rango y el grado de variación genética siendo menor la diversidad génica en la relativamente rango-restringida N. nitida (Premoli 1994, 1997, Premoli et al. 2012a).

La presencia de $N$. nitida sobre las laderas orientales de la Cordillera de Los Andes (Argentina) no ha sido documentada hasta la fecha. Sin embargo, en el sur de Argentina existen áreas con condiciones edáficas y climáticas semejantes a las que la especie se desarrolla en Chile. Por lo tanto, se podría pensar que $N$. nitida podría estar presente al este de Los Andes en áreas donde las condiciones ambientales son propicias para su crecimiento. Se llevó a cabo un estudio genético para confirmar la presencia de $N$. nitida al este de Los Andes y de esa manera descartar la posibilidad que individuos de morfología foliar y arquitectura dudosa no fuera resultado de la plasticidad fenotípica de $N$. dombeyi creciendo en ambientes diferentes, o pertenecientes a distintos rodales o edades. En el presente estudio se informa el hallazgo de los primeros registros de Nothofagus nitida en Argentina, se estima su área de distribución geográfica potencial, y se discute la posibilidad de encontrar individuos de esta especie en otras áreas con similares condiciones climáticas al este de Los Andes.

\section{MÉTODOS}

Sitio de estudio y prospecciones. En dos instancias, en los meses de marzo y abril de 2016, se realizaron salidas de 
prospección biológica a diferentes sitios del fondo del Brazo Sur del lago Menéndez, al oeste del Parque Nacional Los Alerces, provincia de Chubut, Argentina (figura 1A). Durante la primera expedición, se observaron ejemplares aislados de Nothofagus spp. siempreverdes cuyo aspecto no coincidía con ninguna de las especies documentadas para Argentina (figura 1B y D); también llamó la atención la vegetación en las laderas ubicadas al norte del Brazo Sur (figura 1C), que mostraban cambios de coloración del bosque entre pisos altitudinales, con una más clara debajo (perilacustre, presumiblemente correspondiente a N. dombeyi) y otra más oscura sobre la anterior, aparentemente de otro Nothofagus sp. siempreverde. Debido a que las expediciones se realizaron en otoño, se descartó la posibilidad de que estos últimos fueran especies de hoja caduca (lenga o ñire); en sitios cercanos se observó que las mismas presentaban signos de senescencia foliar con hojas color rojo, anaranjado y amarillo. En cambio, por las características morfológicas observadas a nivel de individuo y de hoja, se hipotetizó que dichos individuos podían corresponder a la especie $N$. nitida. Para probar esta premisa, se colectó material foliar (una porción terminal de una rama) de uno de los ejemplares de identidad desconocida ubicado en la desembocadura del río Alerce (MEN1) (figura 1B). Posteriormente, con el objeto de colectar material de nuevos ejemplares putativamente pertenecientes a la especie $N$. nitida, a base de características observables de arquitectura y fisonomía, se recorrieron a pie unos $20 \mathrm{~km}$ en total,

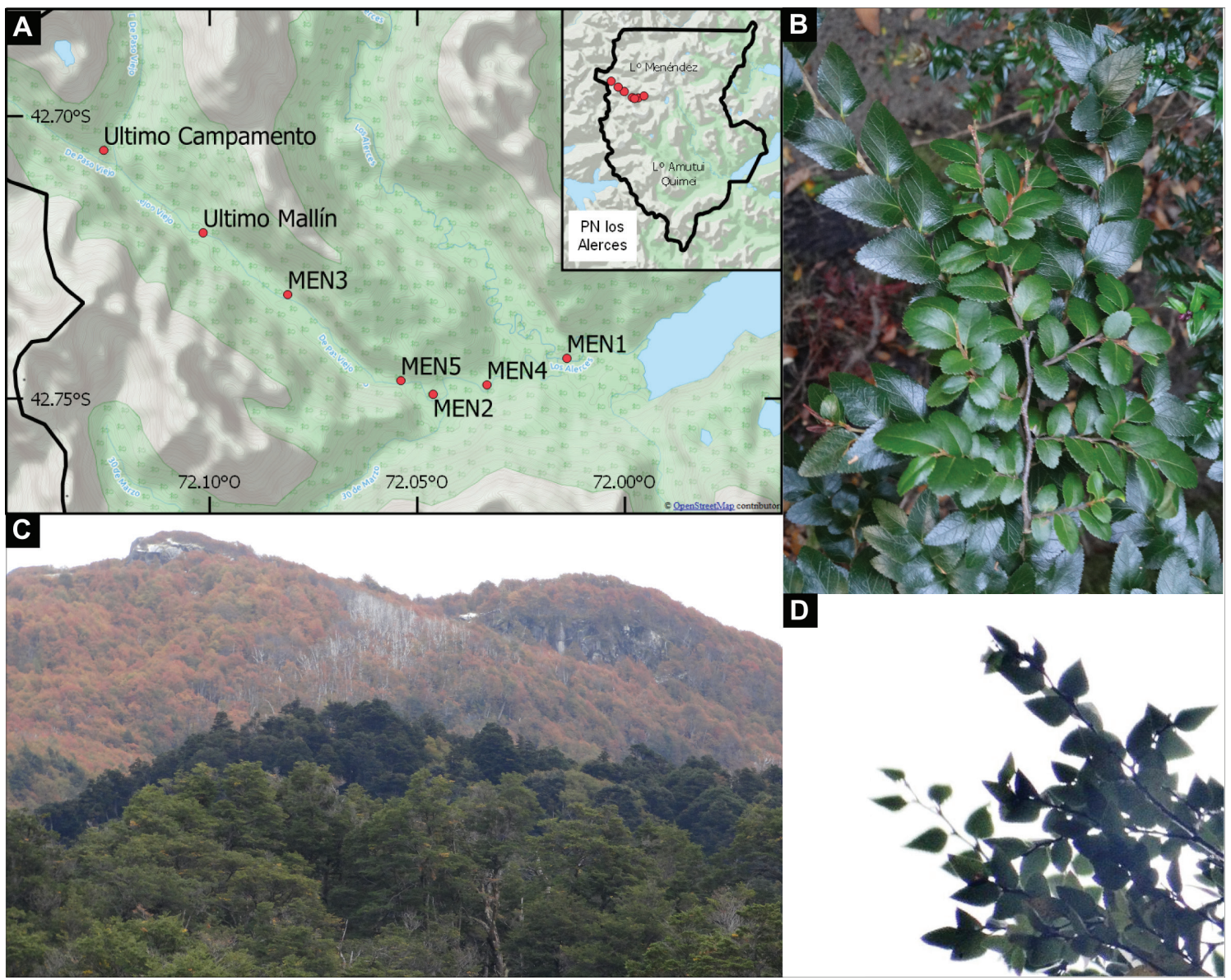

Figura 1. (A) Ubicación de los sitios de colecta a lo largo del margen del río Alerce en el Brazo Sur del lago Menéndez, Parque Nacional Los Alerces, Chubut, Argentina. (B) Fotografía de la rama de uno de los ejemplares colectados de $N$. nitida putativos, superpuesta se encuentra una rama de coihue común ( $N$. dombeyi) para resaltar las diferencias en la morfología de las hojas y la coloración de las mismas. (C) Fotograía del paisaje donde se observa la franja oscura correspondiente -posiblemente- a los individuos de $N$. nitida, la franja más clara correspondiente a $N$. dombeyi, y la franja superior de colores rojizos correspondiente a $N$. pumilio. (D) Detalle del follaje de un coihue de Chiloé del sitio "Último Campamento" (fotos: F. Vidoz).

(A) Location of sampling sites on river banks of Alerce river at the south outlet of Menéndez lake, Los Alerces National Park, Chubut, Argentina. (B) Photograph of a branch of one of the sampled individuals of putative $N$. nitida overlaying a branch of 'coihue' ( $N$. dombeyi) to remark the differences in leaf morphology and coloration. (C) Photograph of the landscape, where the dark green strip possibly corresponds to $N$. nitida individuals, the clearest corresponds to N. dombeyi and the upper reddish one to N. pumilio. (D) Detail of the foliage of 'coihue de Chiloé' at the site "Último Campamento" (Photos: F. Vidoz). 
desde la desembocadura del río Alerce, hasta el denominado Paso Alerce, transitando en general fuera de sendas por el cauce y las orillas del río (figura 1A, cuadro 1).

En el área prospectada, los ejemplares identificados como N. nitida putativos fueron hallados en la orilla norte del río Alerce y del río del Paso Viejo (afluente del primero). No se hallaron ejemplares en sitios expuestos, donde prosperan coihues ( $N$. dombeyi). Las muestras se tomaron de cinco ejemplares de individuos ubicados hacia las menores altitudes ocupadas por un bosque (no visitado por ser inaccesible) cuya coloración a la distancia difería de la de los individuos de $N$. dombeyi. Estos árboles presentaban hojas más oscuras y brillantes y se ubicaban sobre la ladera norte de la boca del río Alerce, sobre el lago Menéndez, entre los 750 y 900 m s.n.m. (figura 1C).

Descripción de los ambientes. La orilla norte del río Alerce y del río del Paso Viejo, corresponden a un ambiente extremadamente húmedo y sombrío (al sur-este de grandes macizos rocosos de la Cordillera de Los Andes). El área del Parque Nacional Los Alerces presenta microclimas de bolsones cálidos como consecuencia de la exposición y altitud, que varía entre $520 \mathrm{~m}$ s.n.m. en los lagos Futalaufquen y Menéndez, y alrededor de 2.000 m\s.n.m. en algunos picos montañosos. Las precipitaciones se presentan según un marcado gradiente Oeste-Este, con valores anuales entre 800-900 mm en el Este y más de $3.000 \mathrm{~mm}$ hacia el Oeste (Monjeau et al. 2006). En el área de estudio, la precipitación y la temperatura media de la estación de crecimiento son de $230 \mathrm{~mm}$ y $12.5^{\circ} \mathrm{C}$, respectivamente, según los datos extraídos de Worldclim (Hijmans et al. 2005) a partir de las coordenadas geográficas de los puntos de muestreo. La matriz general del bosque está compuesta por un primer estrato (emergente) dominado por $N$. dombeyi, un segundo estrato de Laureliopsis philippiana (Looser) Schodde (laurel), Saxegothaea conspicua Lindl. (mañío hembra), y un tercer estrato suprimido compuesto principalmente por grandes ejemplares de Myrceugenia chrysocarpa (O. Berg) Kausel (luma blanca) de 5-6 m de altura. En algunos sectores dominan gigantescos alerces, Fitzroya cupressoides I.M. Johnst de hasta 40 metros de altura. El sotobosque está compuesto principalmente por Chusquea culeou É. Desv. (caña colihue) que se encontraba casi en su totalidad seca, Lomatia ferruginea (Cav.) R.Br. (fuinque), Fuchsia magellanica Lam. (chilco), Azara lanceolata Hook.f. (corcolén) y Desfontainia spinosa Ruiz et Pav. (taique); la liana Hydrangea serratifolia F.Phil (pahueldin), la enredadera Campsidium valdivianum (Phil.) Skottsb. (pilpilvoqui) y Asteranthera ovata Baill. (estrellita), cubren casi enteramente el suelo y los fustes arbóreos, junto a un cojín de musgos y helechos del género Hymenophyllum $\mathrm{Sm}$. (principalmente $H$. pectinatum Nees y Blume, H. tortuosum Hook. y Grev. y $H$. peltatum (Poir.) Desv.). En el sitio Último Campamento (figura 1A) situado a $650 \mathrm{~m}$ s.n.m., el dosel está compuesto por un estrato de individuos de Nothofagus siempreverdes, cuya identidad queremos determinar en este estudio, con un segundo estrato de laurel y Podocarpus nubigenus Lindl. (mañío macho - algunos de gran diámetro), y un tercero de la mencionada luma blanca. El sotobosque está compuesto por ejemplares de caña seca y renovales de laurel, además de las lianas, enredaderas, musgos y helechos anteriormente mencionados.

Análisis de $A D N$. Se colectaron muestras de aproximadamente $20 \mathrm{~cm}$ de largo de la porción terminal de ramas correspondientes a cinco ejemplares de pequeño porte (aproximadamente $5 \mathrm{~m}$ ), a los cuáles se pudo tener acceso (la mayoría de los árboles se encontraban inaccesibles dada la densidad del sotobosque de caña y la irregularidad del terreno). Estos individuos de $N$. nitida putativos fueron hallados bajo la sombra de otros coihues, y otras especies en ambientes ribereños sobre la orilla norte del río Alerce (MEN1) y la orilla orientada al sur de su afluente, el río del Paso Viejo (MEN2-MEN5) (cuadro 1). Para identificar los ejemplares colectados de Nothofagus sp. se utilizaron secuencias de $\mathrm{ADN}$ del espaciador intergénico transcripto, ITS. Este marcador genético corresponde a una región del

Cuadro 1. Coordenadas geográficas de los sitios de colecta de las muestras de Nothofagus sp. en el Parque Nacional Los Alerces, Chubut, Argentina.

Geographic coordinates of the sampling sites of Nothofagus sp in Los Alerces National Park, Chubut, Argentina.

\begin{tabular}{|c|c|c|c|c|}
\hline ID & Latitud (S) & Longitud (O) & $\begin{array}{l}\text { Altitud } \\
\text { (m s.n.m.) }\end{array}$ & Sitio de colecta $\dagger$ \\
\hline MEN1 & $42^{\circ} 44^{\prime} 34,35^{\prime \prime}$ & $72^{\circ} 00^{\prime} 50,31^{\prime \prime}$ & 544 & Río Alerce, brazo sur del lago Menéndez \\
\hline MEN2 & $42^{\circ} 44^{\prime} 57,23^{\prime \prime}$ & $72^{\circ} 02^{\prime} 46,99^{\prime \prime}$ & 562 & Arroyo 30 de Marzo, río del Paso Viejo, brazo sur del lago Menéndez \\
\hline MEN3 & $42^{\circ} 43^{\prime} 53,7^{\prime \prime}$ & $72^{\circ} 04^{\prime} 52,5^{\prime \prime}$ & 585 & Margen norte del río del Paso Viejo, brazo sur del lago Menéndez \\
\hline MEN4 & $42^{\circ} 44^{\prime} 51,52^{\prime \prime}$ & $72^{\circ} 01^{\prime} 59,68^{\prime \prime}$ & 554 & Margen norte del río del Paso Viejo, brazo sur del lago Menéndez \\
\hline MEN5 & $42^{\circ} 44^{\prime} 48,45^{\prime \prime}$ & $72^{\circ} 03^{\prime} 14,31^{\prime \prime}$ & 580 & Margen norte del río del Paso Viejo, brazo sur del lago Menéndez \\
\hline
\end{tabular}

$\dagger$ Todos los individuos fueron identificados a campo y colectados por F. Vidoz. 
ADN nuclear que fue utilizado en estudios previos para resolver las relaciones filogenéticas de las especies del subgénero Nothofagus (Acosta y Premoli 2010), y ha demostrado ser útil ya que las especies puras poseen secuencias diagnósticas que permiten identificarlas (figura 2). La extracción y amplificación de ADN por PCR se realizó siguiendo los protocolos descriptos en Acosta y Premoli (2010). Los productos amplificados fueron enviados para su secuenciación a Macrogen (Seúl, Corea). Las secuencias de ADN obtenidas fueron alineadas y comparadas con secuencias conocidas de las cinco especies del subgénero Nothofagus (las tres especies siempreverdes $N$. betuloides, $N$. dombeyi y N. nitida; y las dos especies de hojas deciduas $N$. antarctica y $N$. pumilio) publicadas anteriormente (Acosta y Premoli 2010). Para establecer las relaciones entre las secuencias analizadas se utilizó el método de agrupamiento del vecino más próximo o "Neighbor-Joining" el cual construye un árbol filogenético a base de la distancia entre los pares de secuencias. Luego de generar el árbol se verificó el soporte de las ramas (clados) que contienen a los individuos $N$. nitida putativos mediante un análisis de remuestreo al azar con reposición (bootstrap) de 500 réplicas. Este método estadístico permite evaluar el nivel de confianza del agrupamiento obtenido en el árbol filogenético. Además, se estimaron las distancias genéticas ( $p$ distance) entre las secuencias de los cinco ejemplares de Nothofagus sp. colectados en este estudio y las secuencias conocidas de las cinco especies del subgénero Nothofagus. Todos los análisis fueron realizados utilizando el programa MEGA6 (Tamura et al. 2013).
Modelo de distribución potencial. A fin de modelar la distribución potencial de la especie y su posible localización al este de Los Andes, se recopiló información sobre la presencia de la especie a partir de distintas fuentes de información. Se utilizaron como datos de presencia distintos puntos con coordenadas de GPS de poblaciones de $N$. nitida que fueron objeto de estudios genéticos previos de la especie en Chile (Premoli 1996a, 1997, Acosta y Premoli 2010, Premoli et al. 2012b, Acosta et al. 2014), información de puntos de ocurrencia de la especie obtenida de la Plataforma Mundial de Información en Biodiversidad (GBIF 2016), y datos de presencia proporcionados por el Herbario de la Universidad de Concepción (Chile) totalizando 140 puntos. Para extraer las condiciones climáticas actuales donde habita $N$. nitida se utilizaron 19 variables bioclimáticas obtenidos de la base de datos WorldClim versión 1 (Hijmans et al. 2005) con una resolución espacial de $30 \mathrm{seg}$. Estos datos son derivados de promedios mensuales de temperatura y precipitación representativos de las dimensiones climáticas relevantes para la determinación de la distribución de especies (Hijmans et al. 2005). Para reducir la autocorrelación espacial entre los puntos de muestreo, aquellos puntos ubicados a menos de $1 \mathrm{~min}$ de latitud fueron removidos del análisis. Se utilizó el programa MAXENT (Phillips et al. 2006) incluyendo las 19 variables bioclimáticas y se seleccionaron las cuatro variables que mejor predicen la distribución potencial. Luego se corrió el programa incluyendo las variables de mayor relevancia, utilizando el $25 \%$ de los puntos para entrenar el modelo, el valor del umbral de convergencia por defec-
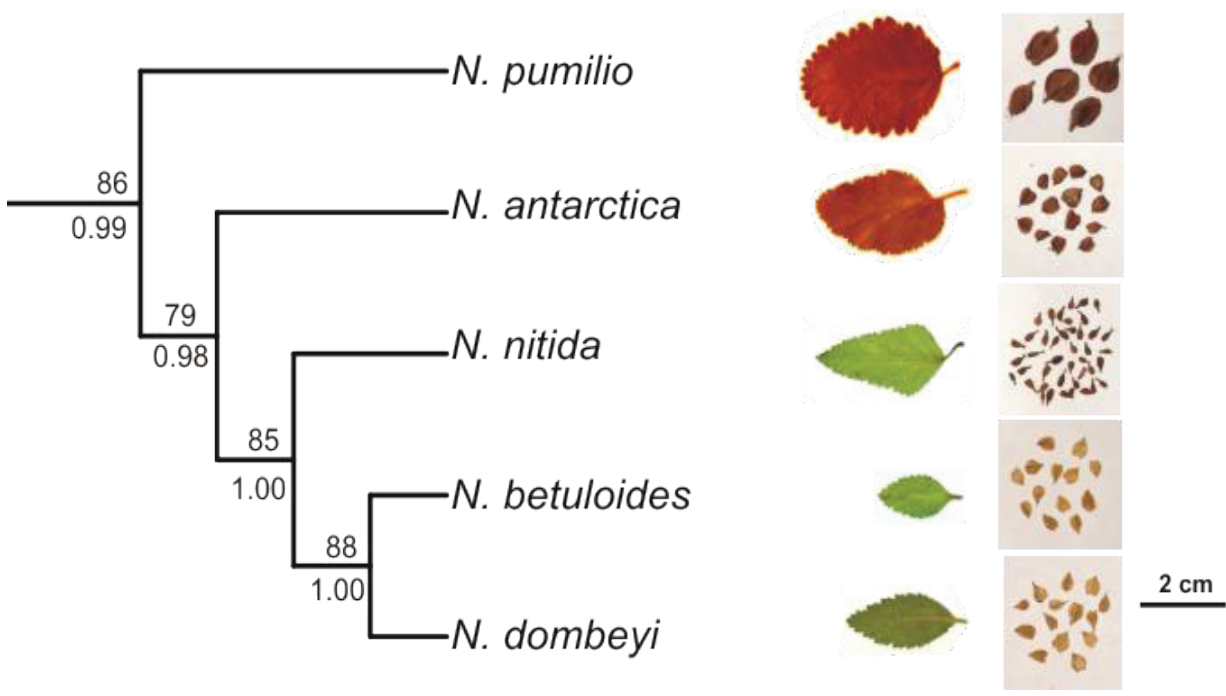

Figura 2. Relaciones filogenéticas de las cinco especies del subgénero Nothofagus basada en secuencias de ADN nuclear (ITS). A modo ilustrativo se incluye la morfología foliar y características de las semillas. Los números indican el soporte de las ramas del análisis de máxima parsimonia (superior) e inferencia Bayesiana (inferior) (extraído de Premoli et al. 2012a).

Phylogenetic relationships of the five species of subgenus Nothofagus based on nuclear DNA sequences (ITS). Leaf morphology and seed characteristics are included. The numbers indicate branch support of the maximum parsimony (above) and Bayesian inference (below) analyses (extracted from Premoli et al. 2012a). 
to $\left(10^{-5}\right)$ y 500 iteraciones. Con los resultados obtenidos a base de las variables climáticas y utilizando el programa QGIS (QGIS Development Team 2016) se elaboró un mapa de la distribución potencial asumiendo que la especie se encuentra en equilibrio con el clima, el que a su vez es el determinante de la distribución de $N$. nitida.

\section{RESULTADOS}

El análisis de las secuencias alineadas de ADN de la región ITS mostraron que cuatro (MEN1, MEN3, MEN4 y MEN5) de los cinco individuos analizados corresponden a la especie $N$. nitida, y el quinto individuo (MEN2) compartía la misma secuencia de ADN con $N$. dombeyi y $N$. betuloides (cuadro 2). Además, el análisis de agrupamiento de Neighbor-Joining de las secuencias de ADN mostró que estos cuatro individuos forman un clado monofilético juntamente con el individuo de $N$. nitida analizado previamente y que fue utilizado en términos de referencia. El quinto individuo $N$. nitida putativo fue incluido en el clado conformado por los individuos de $N$. dombeyi y $N$. betuloides (figura 3). De la misma manera, al comparar las distancias genéticas entre las secuencias de ADN de la región ITS de estos cuatro individuos y las correspondientes secuencias de las cinco especies del subgénero Nothofagus analizadas previamente, se encontraron valores nulos con $N$. nitida, intermedios con las otras dos especies siempreverdes y mayores cuando la comparación se realizó con las dos especies deciduas. Para el individuo MEN2, en cam- bio, la menor distancia genética se observó con $N$. dombeyi y $N$. betuloides (cuadro 3).

Las cuatro variables climáticas con mayor peso para predecir la distribución potencial de $N$. nitida estuvieron relacionadas con la precipitación. Estas fueron: la precipitación media anual, la precipitación del mes más seco, la precipitación del cuartil más seco, y la precipitación del cuartil más frío. El modelo de distribución potencial (figura 4A) realizado para $N$. nitida mostró la existencia de áreas potenciales ubicadas en Argentina en las cuales podría encontrarse la especie. El área de mayor extensión coincidió con los puntos de muestreo donde fueron encontrados los individuos identificados como $N$. nitida en este estudio (figura 4B).

\section{DISCUSIÓN}

Este estudio reporta por primera vez la presencia de individuos de Nothofagus nitida en Argentina, la que hasta ahora era considerada especie endémica de Chile (Rodríguez y Quezada 2003, Donoso et al. 2004, Donoso y Escobar 2006). Los resultados obtenidos mediante el análisis de las secuencias de $\mathrm{ADN}$ permitieron distinguir inequívocamente como $N$. nitida a cuatro de los cinco individuos colectados al este de Los Andes, siendo este el primer registro de la presencia de la especie en Argentina. Sin embargo, el individuo MEN2 que fue colectado como $N$. nitida putativo por sus características morfológicas, fue agrupado con $N$. dombeyi y $N$. betuloides por el análisis genético. El uso de

Cuadro 2. Sitios variables y número de acceso de GenBank de las secuencias de ADN de la región ITS alineadas para las especies de Nothofagus y los individuos de N. nitida putativos colectados en el Brazo Sur del lago Menéndez, Parque Nacional Los Alerces, Chubut, Argentina.

Variable sites and GenBank accession numbers of aligned DNA sequences of the ITS region for Nothofagus species and sampled individuals of putative N. nitida in Los Alerces National Park, Chubut, Argentina.

\begin{tabular}{|c|c|c|c|c|c|c|c|c|c|c|c|c|c|c|c|c|c|c|c|c|c|c|c|}
\hline \multirow{2}{*}{ Especie } & \multirow{2}{*}{$\begin{array}{l}\text { No. de acceso } \\
\text { de GenBank }\end{array}$} & \multicolumn{22}{|c|}{ Sitios polimórficos } \\
\hline & & $\stackrel{n}{n}$ & 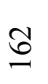 & $\infty$ & $\stackrel{\Xi}{\Xi}$ & $\curvearrowright$ & $\stackrel{\infty}{\vec{\sim}}$ & $\bar{n}$ & $\stackrel{ \pm}{n}$ & 离 & $\begin{array}{l}\infty \\
\infty \\
\infty\end{array}$ & 8 & $\underset{6}{+}$ & $\underset{\sigma}{\infty}$ & તુర & $\frac{2}{6}$ & శ్ర & के & $\vec{g}$ & $\stackrel{\infty}{\sigma}$ & ஜ & $\stackrel{\nabla}{\nabla}$ & $\stackrel{N}{ }$ \\
\hline N. pumilio & GQ863231 & $\mathrm{T}$ & $\mathrm{T}$ & $\mathrm{T}$ & $\mathrm{T}$ & $\mathrm{C}$ & - & $\mathrm{A}$ & - & G & $\mathrm{T}$ & $\mathrm{T}$ & G & $\mathrm{A}$ & G & $\mathrm{C}$ & $\mathrm{T}$ & $\mathrm{A}$ & A & G & $\mathrm{C}$ & $\mathrm{T}$ & $*$ \\
\hline N. antarctica & GQ863233 & $\mathrm{C}$ & $\mathrm{T}$ & $\mathrm{C}$ & $\mathrm{C}$ & $\mathrm{T}$ & - & $\mathrm{T}$ & - & A & $\mathrm{T}$ & $\mathrm{T}$ & $\mathrm{A}$ & A & G & $\mathrm{T}$ & $\mathrm{C}$ & $\mathrm{C}$ & G & $\mathrm{C}$ & $\mathrm{C}$ & $\mathrm{C}$ & - \\
\hline N. betuloides & GQ863244 & $\mathrm{T}$ & $\mathrm{C}$ & $\mathrm{C}$ & $\mathrm{T}$ & $\mathrm{T}$ & $(\mathrm{G})_{2}$ & $\mathrm{~T}$ & $\mathrm{C}$ & A & $\mathrm{C}$ & A & G & G & $\mathrm{T}$ & $\mathrm{T}$ & $\mathrm{C}$ & $\mathrm{C}$ & G & $\mathrm{C}$ & $\mathrm{T}$ & $\mathrm{C}$ & - \\
\hline N. dombeyi & GQ863234 & $\mathrm{T}$ & $\mathrm{C}$ & $\mathrm{C}$ & $\mathrm{T}$ & $\mathrm{T}$ & $(G)_{2}$ & $\mathrm{~T}$ & $\mathrm{C}$ & A & $\mathrm{C}$ & A & G & G & $\mathrm{T}$ & $\mathrm{T}$ & $\mathrm{C}$ & $\mathrm{C}$ & $\mathrm{G}$ & $\mathrm{C}$ & $\mathrm{T}$ & $\mathrm{C}$ & - \\
\hline N. nitida & GQ863240 & $\mathrm{T}$ & $\mathrm{T}$ & $\mathrm{C}$ & $\mathrm{T}$ & $\mathrm{T}$ & - & $\mathrm{T}$ & - & A & $\mathrm{T}$ & A & G & G & $\mathrm{T}$ & $\mathrm{T}$ & $\mathrm{T}$ & $\mathrm{C}$ & G & $\mathrm{C}$ & $\mathrm{T}$ & $\mathrm{C}$ & - \\
\hline MEN1 & KX620857 & $\mathrm{T}$ & $\mathrm{T}$ & $\mathrm{C}$ & $\mathrm{T}$ & $\mathrm{T}$ & - & $\mathrm{T}$ & - & $\mathrm{A}$ & $\mathrm{T}$ & A & G & G & $\mathrm{T}$ & $\mathrm{T}$ & $\mathrm{T}$ & $\mathrm{C}$ & G & $\mathrm{C}$ & $\mathrm{T}$ & $\mathrm{C}$ & - \\
\hline MEN2 & KX620858 & $\mathrm{T}$ & $\mathrm{C}$ & $\mathrm{C}$ & $\mathrm{T}$ & $\mathrm{T}$ & $(\mathrm{G})_{2}$ & $\mathrm{~T}$ & $\mathrm{C}$ & A & $\mathrm{C}$ & A & G & G & $\mathrm{T}$ & $\mathrm{T}$ & $\mathrm{C}$ & $\mathrm{C}$ & G & $\mathrm{C}$ & $\mathrm{T}$ & $\mathrm{C}$ & - \\
\hline MEN3 & KX620859 & $\mathrm{T}$ & $\mathrm{T}$ & $\mathrm{C}$ & $\mathrm{T}$ & $\mathrm{T}$ & - & $\mathrm{T}$ & - & A & $\mathrm{T}$ & A & G & G & $\mathrm{T}$ & $\mathrm{T}$ & $\mathrm{T}$ & $\mathrm{C}$ & G & $\mathrm{C}$ & $\mathrm{T}$ & $\mathrm{C}$ & - \\
\hline MEN4 & KX620860 & $\mathrm{T}$ & $\mathrm{T}$ & $\mathrm{C}$ & $\mathrm{T}$ & $\mathrm{T}$ & - & $\mathrm{T}$ & - & A & $\mathrm{T}$ & A & G & G & $\mathrm{T}$ & $\mathrm{T}$ & $\mathrm{T}$ & $\mathrm{C}$ & G & $\mathrm{C}$ & $\mathrm{T}$ & $\mathrm{C}$ & - \\
\hline MEN5 & KX620861 & $\mathrm{T}$ & $\mathrm{T}$ & $\mathrm{C}$ & $\mathrm{T}$ & $\mathrm{T}$ & - & $\mathrm{T}$ & - & A & $\mathrm{T}$ & $\mathrm{A}$ & $\mathrm{G}$ & $\mathrm{G}$ & $\mathrm{T}$ & $\mathrm{T}$ & $\mathrm{T}$ & $\mathrm{C}$ & G & $\mathrm{C}$ & $\mathrm{T}$ & $\mathrm{C}$ & - \\
\hline
\end{tabular}

* Inserción de 5 pb (“GCATT”) en N. pumilio. 


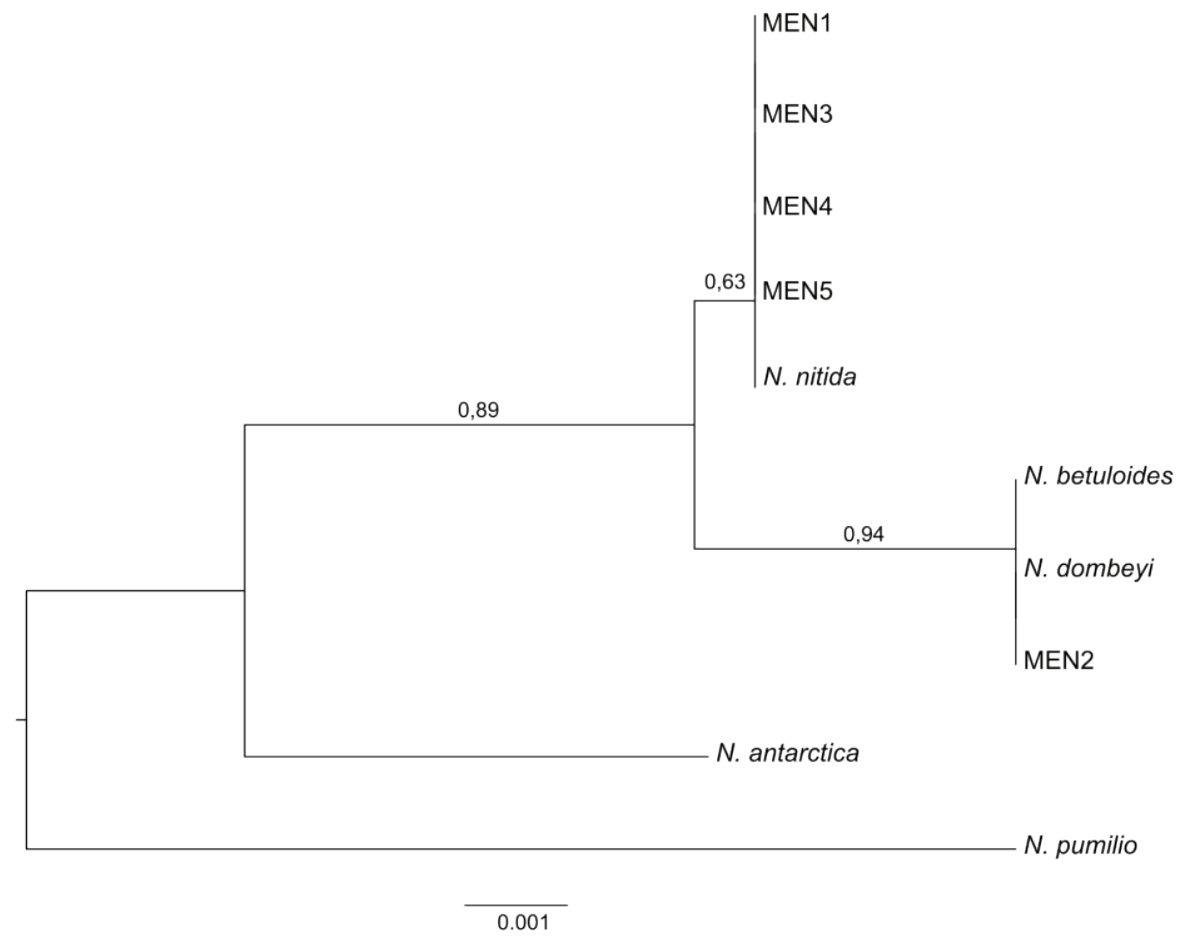

Figura 3. Árbol de agrupamiento (Neighbor-Joining) de los individuos N. nitida putativos y las especies puras del subgénero Nothofagus basado en los sitios variables de la región ITS del ADN nuclear. Los números indican el soporte de las ramas que contienen las secuencias de los individuos analizados del Parque Nacional Los Alerces, Chubut, Argentina.

Neighbor-Joining tree of the putative individuals of $N$. nitida and pure species of subgenus Nothofagus based on the proportion of variable sites of the nuclear DNA ITS region. The numbers indicate bootstrap support values of the branches which contain sequences of individuals from Los Alerces National Park, Chubut, Argentina.

Cuadro 3. Distancia genética (p-distance) entre secuencias de ADN de la región ITS de las cinco especies del subgénero Nothofagus y las muestras colectadas en el Brazo Sur del lago Menéndez, Parque Nacional Los Alerces, Chubut, Argentina. " Distancias genéticas nulas entre pares de individuos, en particular los de $N$. nitida putativos $\left(^{*}\right)$ cuya identidad corresponde a especies puras.

Genetic distance ( $p$-distance) among DNA sequences of the ITS region of the five species of subgenus Nothofagus and collected samples in Los Alerces National Park, Chubut, Argentina. " Similar genetic distances among individuals, mainly among putative $N$. nitida $\left({ }^{*}\right)$ individuals which correspond to pure species.

\begin{tabular}{|c|c|c|c|c|c|c|c|c|c|}
\hline & $N p$ & $N b$ & $N d$ & $N n$ & MEN1* & MEN2 & MEN3* & MEN4* & MEN5* \\
\hline N. antarctica & 0,016 & 0,011 & 0,011 & 0,010 & 0,010 & 0,011 & 0,010 & 0,010 & 0,010 \\
\hline N. pumilio & & 0,020 & 0,020 & 0,016 & 0,016 & 0,020 & 0,016 & 0,016 & 0,016 \\
\hline N. betuloides & & & $0,000^{*}$ & 0,004 & 0,004 & $0,000^{\#}$ & 0,004 & 0,004 & 0,004 \\
\hline N. dombeyi & & & & 0,004 & 0,004 & $0,000^{\#}$ & 0,004 & 0,004 & 0,004 \\
\hline N. nitida & & & & & $0,000^{\#}$ & 0,004 & $0,000^{\#}$ & $0,000^{\#}$ & $0,000^{\#}$ \\
\hline MEN1* & & & & & & 0,004 & $0,000^{*}$ & $0,000^{*}$ & $0,000^{\#}$ \\
\hline MEN2 & & & & & & & 0,004 & 0,004 & 0,004 \\
\hline MEN3* & & & & & & & & $0,000^{\#}$ & $0,000^{\#}$ \\
\hline MEN4* & & & & & & & & & $0,000^{*}$ \\
\hline
\end{tabular}




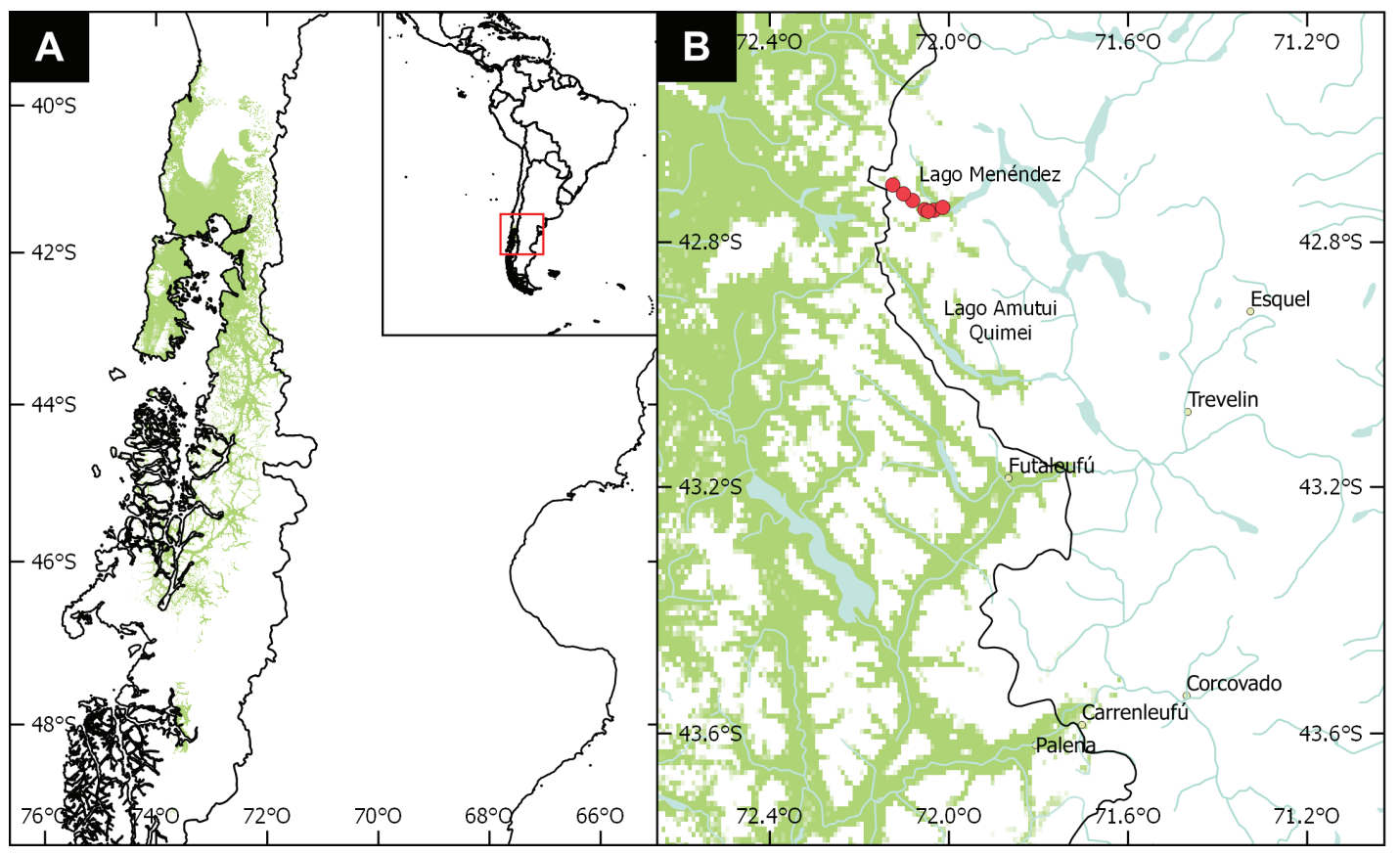

Figura 4. (A) Mapa de la distribución potencial de la especie N. nitida modelada con MAXENT. (B) Detalle del área de distribución potencial para la especie en Argentina y parte de Chile. Los puntos rojos indican los sitios de colecta de ejemplares de $N$. nitida putativos en el Brazo Sur del lago Menéndez, Parque Nacional Los Alerces, Chubut, Argentina.

(A) Species potential distribution model of $N$. nitida obtained with MAXENT. (B) Detail of the potential distribution area of the species in Argentina and part of Chile. The red dots indicate sampling sites of putative N. nitida at the south outlet of Menendez Lake, Los Alerces National Park, Chubut, Argentina.

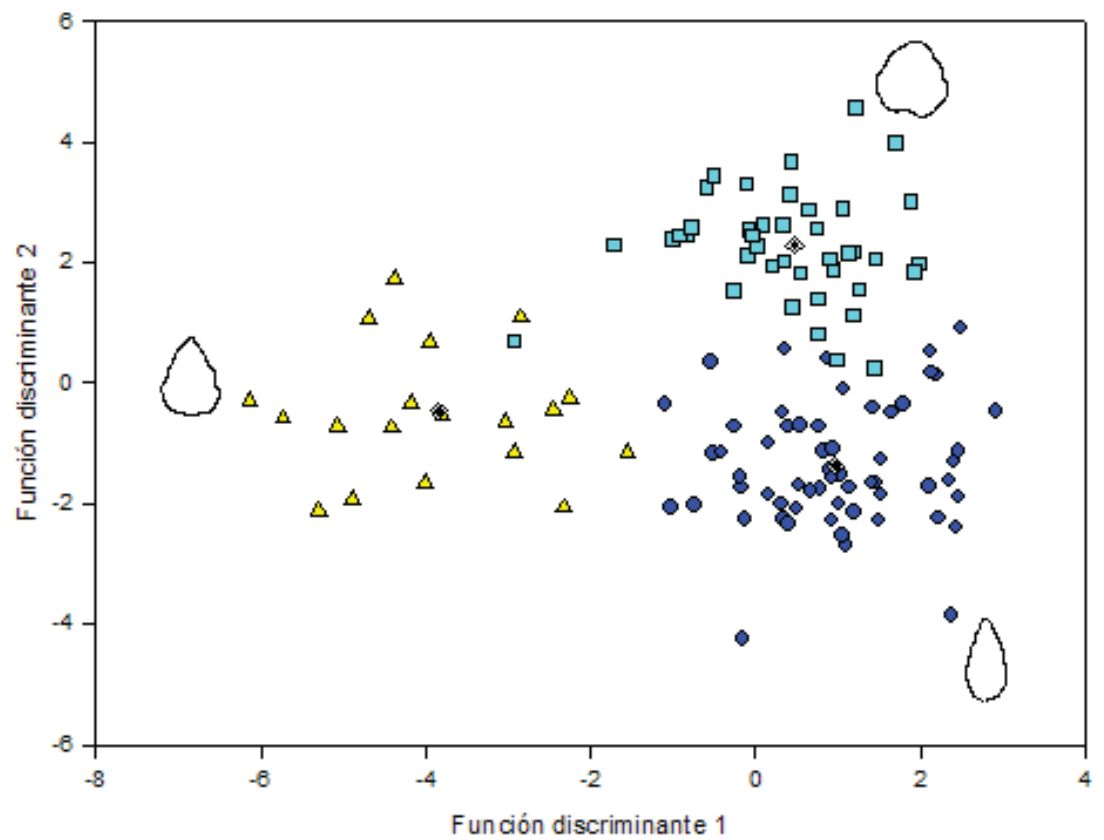

Figura 5. Análisis discriminante de la variación en la arquitectura foliar de Nothofagus siempreverdes sudamericanos. Se representan las hojas con morfologías más extremas de $N$. dombeyi, $N$. betuloides y $N$. nitida. El centroide de cada grupo se indica con un símbolo romboidal. Triángulos amarillos: $N$. nitida, cuadrados celestes: $N$. betuloides, rombos azules: $N$. dombeyi (extraído de Premoli 1996b).

Discriminant analysis of leaf architecture variation of South American evergreen Nothofagus species. Leaves with extreme morphological features of $N$. dombeyi, $N$. betuloides and $N$. nitida are represented. The centroid of each group is indicated by a rhomboidal symbol. Yellow triangles: $N$. nitida, light blue squares: $N$. betuloides, and blue rhomboids: N. dombeyi (extracted from Premoli 1996b). 
herramientas genéticas como ser secuencias del ADN nuclear que se encuentra altamente conservado en individuos de una misma especie permitió confirmar la identidad de los mismos. Esto es particularmente relevante en el caso de los Nothofagus siempreverdes cuya identificación a campo se basa en caracteres foliares y florales que presentan variación cuantitativa y elevada variabilidad fenotípica. Si bien el número de individuos analizados es acotado, no son individuos adultos, y se encontraban levemente distanciados del estrato de árboles con hojas más oscuras y brillantes observados en la ladera ubicada más al norte en la boca del río Alerce, estos podrían ser producto de la dispersión de un rodal semillero próximo a los individuos muestreados. Dado que la dispersión de las semillas de la especie ocurre a distancias cortas (Veblen et al. 1996), sería poco probable que los juveniles muestreados sean producto de eventos de dispersión a larga distancia desde poblaciones ubicadas al oeste de Los Andes, en Chile. Se requieren estudios poblacionales para analizar el grado de flujo génico y reconstruir la historia biogeográfica de las poblaciones de $N$. nitida sobre ambas vertientes de Los Andes.

Estudios realizados previamente muestran evidencia genética (Premoli 1996a, 1997, Acosta y Premoli 2010), ecofisiológica (Premoli 1994) y morfológica (Premoli 1996b) que permite diferenciar a $N$. nitida de las otras dos especies siempreverdes, $N$. betuloides y $N$. dombeyi (figuras 2, 3 y 5). Estudios realizados en plántulas coetáneas de $N$. nitida cultivadas en condiciones ambientales homogéneas muestran que las diferencias morfológicas y ecofisiológicas encontradas con las otras dos especies siempreverdes poseen base genética (Premoli 1994, Premoli 1996b). Por otro lado, estudios poblacionales a base de isoenzimas mostraron que $N$. nitida posee cuatro alelos que no están presentes en las otras dos especies siempreverdes y que, por lo tanto, podrían considerarse diagnósticos (Premoli 1996a, 1997). Además, $N$. nitida mostró un alelo fijado para tres loci monomórficos diferente al encontrado en $N$. betuloides y $N$. dombeyi (ver figura 1 en Premoli 1996a). La identidad genética promedio de las poblaciones de $N$. nitida respecto a las otras dos especies siempreverdes resultó $<0,7$; mientras que la similitud entre las poblaciones de $N$. betuloides y $N$. dombeyi fue $>0,9$ (Premoli 1996a, 1997). Esta evidencia muestra que N. nitida es una unidad taxonómica claramente distinguible genéticamente de las otras dos especies siempreverdes y que si bien puede hibridar con ellas (Donoso y Atienza 1983), ha mantenido su identidad a lo largo del tiempo debido a que habita condiciones ambientalmente diferentes a $N$. betuloides y N. dombeyi (Premoli 1996b, Premoli et al. 2012a). En particular, N. nitida está restringida a sitios superhúmedos y/o suelos frecuentemente mal drenados con precipitaciones constantes y superiores a $2000 \mathrm{~mm}$ por año (Piper 2007, Piper et al. 2007). En cambio, N. dombeyi puede crecer sobre las laderas orientales de Los Andes bajo regímenes de menor precipitación $\mathrm{y}$, por lo tanto, se podría considerar relativamente más tolerante a la sequía que $N$. nitida (Piper et al. 2007). Por otro lado, $N$. betuloides habita en sitios de mayor altitud y latitud, lo que pueden resultar en una mayor tolerancia de esta especie a las bajas temperaturas que $N$. nitida y N. dombeyi (Premoli 1994).

Los ejemplares colectados de $N$. nitida que fueron hallados a lo largo de la línea de prospección de sentido SurOeste se encontraban, en general, cercanos a ejemplares de mañío macho, varios de gran porte. Esta es una asociación muy común (característica y extensa) dentro del Bosque Laurifolio de Chiloé, que representa la situación más húmeda (hidrófita) de la región del Bosque Laurifolio (sistema de clasificación de Gajardo 1994), que se distribuye en suelos planos de mal drenaje. Tal asociación forestal es dominante en el Parque Pumalín (Gastó Coderach et al. 2000), siendo notable la similitud en la cobertura vegetal del área prospectada, con la de ese Parque transandino. El hecho que $N$. nitida ocurra sobre las vertientes orientales de la Cordillera de Los Andes, sugiere que los límites de distribución de esta especie requieren ser revisados. El modelo de distribución realizado para la especie muestra que es posible que existan más individuos y/o poblaciones al este de la Cordillera de Los Andes. Se destaca la importancia de estudiar las características genéticas de estos individuos y/o poblaciones ubicados hacia el límite oriental de la distribución. Los márgenes de distribución son relevantes no solamente por encontrarse alejados del centro del rango de las especies y, por lo tanto, sufrir limitaciones al flujo génico con otras poblaciones, sino que además podrían estar sujetos a regímenes diferenciales de selección por estar bajo condiciones ambientales distintas a las encontradas en el centro del rango. Dichas poblaciones podrían contener potenciales novedades evolutivas bajo situaciones ambientales no habituales, $y$, por lo tanto, valiosas bajo escenarios de cambio climático (por ejemplo, mostrar resistencia al estrés hídrico), las cuales, además, podrían transmitirse a otras especies del subgénero Nothofagus por hibridación/ introgresión (Premoli 1996a, Acosta y Premoli 2010, Premoli et al. 2012a, Acosta et al. 2014). Las especies del subgénero Nothofagus son linajes antiguos que han desarrollado adaptaciones a lo largo de su historia evolutiva y, por lo tanto, tendrían el potencial de responder a cambios en el clima (Premoli et al. 2012a). Por otro lado, se ha demostrado para varias especies que ciertas zonas al este de Los Andes habrían funcionado como refugios glaciales en los que se habrían preservado variantes genéticas únicas de relevancia en conservación. Tal es el caso de poblaciones en el extremo sur de la distribución del alerce en el sur-este de Argentina, que es considerado un reservorio de diversidad genética para la especie (Premoli et al. 2000).

\section{CONCLUSIONES}

El hallazgo aquí reportado permite confirmar mediante el uso de herramientas genéticas la presencia de Nothofagus nitida en Argentina. Además, el modelaje de la distribución potencial realizado a base de las condiciones 
climáticas favorables para el crecimiento de la especie muestra ser una herramienta importante para poder determinar los límites del área de distribución de las especies, que después deben ser corroboradas a campo. Se destaca la relevancia de realizar estudios de prospección botánica en áreas remotas sobre la Cordillera de Los Andes a fin de revisar los límites de distribución de $N$. nitida, y de otras especies que comparten su hábitat, en zonas donde aún no han sido reportadas.

\section{AGRADECIMIENTOS}

Al Guardaparque A. L. Rodriguez Albertani (Jefe del Dpto. Protección y Guardaparques) por su colaboración en la logística, y a los Guardaparques: M. Guisasola, G. Paramosz, P. Vargas, L. Machado y B. Ramos, del Parque Nacional Los Alerces, por su valiosa colaboración en el terreno. A dos revisores anónimos por los comentarios realizados que sirvieron para mejorar la versión final de este trabajo. El material de Nothofagus spp. se colectó en uso de los permisos $\mathrm{N}^{\circ} 727 / 06$ y No840/08 otorgados por la Administración de Parques Nacionales. Financiamiento: PICT 2012-0688; PICT 2013-2404. ACP, MCA, PM, SI y VSO son miembros del CONICET.

\section{REFERENCIAS}

Acosta MC, AC Premoli. 2010. Evidence of chloroplast capture in South American Nothofagus (subgenus Nothofagus, Nothofagaceae). Molecular Phylogenetics and Evolution 54: 235-242.

Acosta MC, P Mathiasen, AC Premoli. 2014. Retracing the evolutionary history of Nothofagus in its geo-climatic context: new developments in the emerging field of phylogeology. Geobiology 12: 497-510.

Donoso Z C, J Atienza H. 1983. Hibridación natural entre especies de Nothofagus siempreverdes en Chile. Bosque 5(1): 21-34.

Donoso Z C, B Escobar. 2006. Nothofagus nitida (Phil.) Krasser. Coigüe de Chiloé, Coigüe. In Donoso Z C ed. Las especies arbóreas de los bosques templados de Chile y Argentina. Autoecología. Valdivia, Chile. Marisa Cuneo Ediciones. p. 462-470.

Donoso Z C, AC Premoli, P Donoso. 2004. Variación en Nothofagus siempreverdes Sudamericanos. In Donoso Z C, AC Premoli, L Gallo, R Ipinza eds. Variación intraespecífica en las especies arbóreas de los bosques templados de Chile y Argentina. Santiago, Chile. Editorial Universitaria. p. 189213.

Gajardo R. 1994. La vegetación natural de Chile, clasificación y distribución geográfica. Santiago, Chile. Editorial Universitaria. $165 \mathrm{p}$.

Gastó Coderach, J, A Retamal Masferrer, D Guzmán Fuentes. 2000. Proyecto Pumalín, Informe técnico Santuario de la Naturaleza. Santiago, Chile. Pontificia Universidad Católica. $178 \mathrm{p}$.

GBIF (Global Biodiversity Information Facility, DK). 2016. GBIF Occurrence Download. Consultado 23 jun. 2016.
Disponible en http://doi.org/10.15468/dl.pelxen.

Hijmans RJ, SE Cameron, JL Parra, PG Jones, A Jarvis. 2005. Very high resolution interpolated climate surfaces for global land areas. International Journal of Climatology 25: 1965-1978.

Hill RS, J Read. 1991. A revised infrageneric classification of Nothofagus (Fagaceae). Botanical Journal of the Linnean Society 105: 37-72.

Manos PS. 1997. Systematics of Nothofagus (Nothofagaceae) based on rDNA spacer sequences (ITS): taxonomic congruence with morphology and plastid sequences. American Journal of Botany 84(9): 1137-1155.

Monjeau A, S Nazar Anchorena, MV Fernandez Montoni, J Marquez, D Alcalde, A Diorio, H Galván, C Denholm, A Di Vicenzo, FA Gonzalez. 2006. Perfil de Área Protegida Argentina: Parque Nacional Los Alerces. Diagnóstico del estado de conservación en áreas protegidas. 44 p. (Informe Técnico, ParksWatch).

Phillips SJ, RP Anderson, RE Schapire. 2006. Maximum entropy modeling of species geographic distributions. Ecological Modelling 190: 231-259.

Piper FI. 2007. Rasgos de Nothofagus dombeyi y N. nitida vinculados al desempeño en gradientes de disponibilidad hídrica y lumínica: consistencia con sus patrones de distribución. Tesis Doctoral. Concepción, Chile. Facultad de Ciencias Naturales y Oceanográficas, Universidad de Concepción. $112 \mathrm{p}$.

Piper FI, LJ Corcuera, M Alberdi, C Lusk. 2007. Differential photosynthetic and survival responses to soil drought in two evergreen Nothofagus species. Annals of Forest Science 64: 447-452.

Premoli AC. 1994. Variación genética, morfológica y ecofisiológica en una especie de Nothofagus geográficamente restringida y dos ampliamente distribuidas del Sur de Sudamérica. Tesis Doctoral. Boulder, Estados Unidos. Departamento de Biología, Universidad de Colorado. 148 p.

Premoli AC. 1996a. Allozyme polymorphisms, outcrossing rates, and hybridization of South American Nothofagus. Genetica 97: 55-64.

Premoli AC. 1996b. Leaf architecture of South American Nothofagus (Nothofagaceae) using traditional and new methods in morphometrics. Botanical Journal of the Linnean Society 121: 25-40.

Premoli AC. 1997. Genetic variation in a geographically restricted and two widespread species of South American Nothofagus. Journal of Biogeography 24: 883-892.

Premoli AC, T Kitzberger, TT Veblen. 2000. Isozyme variation and recent biogeographical history of the long-lived conifer Fitzroya cupressoides. Journal of Biogeography 27: 251-260.

Premoli AC, MC Acosta, P Mathiasen, C Donoso Z. 2012a. Variación genética en Nothofagus (subgénero Nothofagus). Bosque 33(2): 115-125.

Premoli AC, P Mathiasen, MC Acosta, VA Ramos. 2012b. Phylogeographically concordant chloroplast DNA divergence in sympatric Nothofagus s.s. How deep can it be? New Phytologist 193: 261-275.

QGIS Development Team. 2016. QGIS Geographic Information System. Open Source Geospatial Foundation Project. Consultado 4 oct. 2016. Disponible en http://www.qgis.org

Rodríguez R, M Quezada. 2003. Berberidaceae-Betulaceae. In 
Marticorena C, R Rodríguez eds. Flora de Chile Vol. 2(2). Concepción, Chile. Universidad de Concepción. p. 64-76.

Tamura K, G Stecher, D Peterson, A Filipski, S Kumar. 2013. MEGA6: molecular evolutionary genetic analysis version 6.0. Molecular Biology and Evolution 30: 2725-2729.
Veblen TT, C Donoso Z, T Kitzberger, AJ Rebertus. 1996. Ecology of Southern Chilean and Argentinean Nothofagus Forests. In Veblen TT, RS Hill, J Read eds. The ecology and Biogeography of Nothofagus Forests. New Heaven and London, UK. Yale University Press. p. 293-353.

Recibido: 05.10.16

Aceptado: 12.06.17 
